\title{
Ermittlung des monetären Werteinventars als Basis von Analysen naturgefahreninduzierter Risiken in Tirol (Österreich)
}

\author{
Matthias Huttenlau, Johann Stötter, Innsbruck
}

\section{Einleitung}

In Zeiten des globalen Wandels ist eine deutliche Zunahme der mit Naturgefahrenprozessen verbundenen Risiken zu beobachten. Damit die Gesellschaft diesen Herausforderungen adäquat begegnen kann, ist eine repräsentative Bestimmung des relevanten Werteinventars, die so genannten Risikoelemente, im Rahmen von Risikountersuchungen von zentraler Bedeutung. Diese Untersuchungen haben dabei das Ziel, negative Folgen eines potenziell gefährlichen Naturprozesses (Douglas 2007) durch standardisiert konzeptionelle Ansätze (z.B. BorTER 1999; HeINIMANN et al. 1998; Hollenstein 1997; Kienholz 2005; WiLHELM 1997) zu ermitteln. Das Risiko lässt sich hierbei als ein Produkt aus Gefahr, Risikoelementen und Vulnerabilität beschreiben (BoHLE \& GLADE 2007; KLEIST 2006). Dabei kann entsprechend dem Detaillierungsgrad sowie der Größe des Untersuchungsgebietes zwischen mikro-, meso- und makroskaligen (z.B. Bund DER INGENIEURE FÜr WASSERWIRTSCHAFT, ABFALLWIRTSchaft und Kulturbau (BWK) 2001; Gewalt et al. 1996) bzw. lokalen und regionalen Maßstabsebenen (z.B. Glade \& Crozier 2005; Heinimann et al. 1998; HollensteIn 1997) unterschieden werden. Ein skalenübergreifender Ansatz wird von BORTER (1999) diskutiert.

Im Gegensatz zur umfangreichen Literatur über Methoden der Gefahren- und physischen Vulnerabilitätsanalyse sind nur wenige Studien mit einer expliziten Methodenbeschreibung zur Quantifizierung und Monetarisierung der Risikoelemente (KLEIST et al. 2006: 542) bekannt. Auf regionaler Ebene erfolgt die Quantifizierung mittels digitaler Pläne und Daten der amtlichen Statistiken (z.B. IKoNE - INTEGRIERENDE KonzePtion Neckar-Einzugsgebiet 2003; KeILER et al. 2006; MeYer 2005; Ministerium FÜr UMWelt, RAUMORDNUNG UND LANDWIRTSCHAFT NORDRHEIN-WESTFALEN (MURL) 2000), die durch Flächennutzungsinformationen (z.B. IKoNE 2003) und Geomarketingdaten (MeYer 2005) ergänzt werden können. Die Monetarisierung von Gebäudewerten erfolgt generell mittels durchschnittlicher Versicherungssummen (z.B. MEYER 2005; MURL 2000, ) oder über flächenbezogene Basiswerte (IKoNE 2003). Bei lokalen Analysen werden die Risikoelemente aus Geodaten abgeleitet, durch Methoden der Fernerkundung sowie Kartierungen konkretisiert und mit Daten der amtlichen Statistik komplettiert (z.B. Borter 1999; HeINIMANN et al. 1998; Keiler 2004; Keiler et al. 2004; Reese \& Markau 2004). Die Ermittlung des Gebäudewertes erfolgt über mittlere, nach Objektfunktionen differenzierte Werte für einzelne Gebäude (Fuchs et al. 2004), Grund- bzw. Nutzflächen- (Merz 2006; ReEse \& MARKAU 2004) oder Volumeneinheiten (KeILER 2004; KeILER et al. 2004), aber auch sehr detailliert anhand der so genannten Normalherstellungskosten (KLEIST et al. 2006; REESE $\&$ MARKAU 2004). Die Inventarwerte (sofern nicht bereits im Gebäudewert inkludiert) werden über mittlere Versicherungssummen (REESE \& MARKAU 2004) ermittelt. Heinimann et al. (1998), Borter (1999) und das Bundesamt für Wasser Und Geologie (BWG, 2005) wenden einen generellen Indikatorenansatz an. Hamann \& Reese (2000) sowie Reese \& Markau (2004) konnten in einem Methodikvergleich in Schleswig-Holstein für unterschiedliche Risikoindikatoren wie etwa Gebäude- und Inventarwerte einen sehr hohen Differenzfaktor zwischen Bearbeitungen mit Methoden der Meso- und Mikroebene konstatieren. Dies beeinträchtigt in weiterer methodischer Folge das Ergebnis risikobasierter Analysen wesentlich. Kraftfahrzeugen (Kfz) werden ermittelte durchschnittliche Werte zugewiesen (z.B. KeILER 2004; REeSE \& MARKAU 2004). Die monetäre Bewertung des äußerst sensiblen Faktors menschlichen Lebens (Merz 2006) ist nach KoK et al. (2004) aus ethisch-moralischen Gründen nicht zulässig. Anderweitige Studien erfassen diesen Faktor über die indirekten Methoden des Humankapital- (Fuchs \& McAlpin 2005) bzw. des willingnessto-pay- Ansatzes (LeITer 2006; Merz 2006).

Die Quantifizierung und Monetarisierung potenzieller Risikoelemente, das für die Risikoindikatoren Gebäude-, Inventar- und Kfz-Werte sowie menschliches Leben integrale Werteinventar im Untersuchungsgebiet, sind Bestandteil der vorgestellten Arbeit. Diese verfolgt einen objektorientierten versicherungstechnischem Ansatz, bei dem das Hauptaugenmerk auf Gebäude- und Inventarwerten liegt. Das Attribut «versicherungstechnisch» beschreibt hierbei den betriebswirtschaftlichen Charakter der ermittelten und angewandten Werte auf Basis von am Markt gehandelten Versicherungssummen, was im Vergleich mit volkswirtschaftlichen Ansätzen zu berücksichtigen ist. Untersuchungsgebiet ist das österreichische Bundesland Tirol. Entsprechend der Zielsetzung und der Größe des Untersuchungsgebietes liegt der Bearbeitungsmaßstab der Studie auf der regionalen Ebene, 
wobei, unter Berücksichtigung methodischer Ansätze der lokalen Ebene, das Ziel verfolgt wird, die angesprochenen Differenzfaktoren auf ein akzeptables Maß zu reduzieren. Angesichts des komplexen und unscharfen Verhaltens immobiler Risikoelemente werden die Kategorien Kfz und Personen statisch betrachtet und besitzen demnach für diese Analyse einen immobilen Charakter. Für den Aktualitätsstand der Daten kann, wenn nicht anders erwähnt, das Jahr 2006 angeführt werden.

\section{Methoden und Daten zur monetären Quantifizierung der Risikoelemente}

\subsection{Identifizierung, Quantifizierung, Charakterisie- rung und Verortung der Risikoelemente}

Die Digitale Katastralmappe (DKM) stellt die von den Vermessungsämtern geführte lagegenaue Situation im Untersuchungsgebiet dar, ihr ist aber ein nicht unwesentliches Aktualisierungs- und Nachführungsdefizit immanent (vgl. KeILER et al. 2006). Unabhängig davon führt das Amt der Tiroler Landesregierung einen aus Farborthofotos extrahierten GebäudeLayer, der synchron mit einem flächendeckend geokodierten Adressdatensatz aktualisiert wird. Dieser Datensatz besitzt keine geodätische Lagegenauigkeit und differenziert infolge der automatisierten Erstellung einzelne, jedoch gemeinsam überbaute Gebäude (z.B. Reihenhäuser) nicht.

Alle eindeutig identifizierbaren Adresskoordinaten (Straßenname und Hausnummer) des Adressdatensatzes, in weiterer Folge als Risikoadressen bezeichnet, wurden mit dem Gebäude-Layer sowie mit aus der DKM extrahierten Gebäudeflächen verschnitten. Adresspunkte mit einem maximalen Abstand von 2 Metern zu entsprechenden Gebäudeflächen werden in die Zielfläche lageverschoben. Adresspunkten ohne Gebäude, das zugeordnet werden konnte, wurde ein Kreis als Repräsentant des Gebäudes zugewiesen, der der mittleren Gebäudegrundfläche der relevanten Gemeinde entspricht.

Zur Ermittlung und Differenzierung der spezifischen Objektfunktion eignet sich der aus dem Flächenwidmungsplan abgeleitete konkrete Verwendungszweck der einzelnen Grundflächen. In Tirol werden derzeit die Flächenwidmungspläne überarbeitet und neu erlassen, wobei bis Juli 2007 für 85 Prozent der Gemeinden entsprechend revidierte Flächenwidmungspläne vorlagen. Die in den Flächenwidmungsplänen angeführten detaillierten Widmungskategorien wurden zu sechs übergeordneten Kategorien zusammengefasst: (1) Wohnen, (2) gemischte Nutzung, (3) Landwirtschaft, (4) Freiland, (5) Gewerbe/Industrie sowie (6) Tourismus. Für Gemeinden ohne aktuellen Flächen- widmungsplan konnten die in der DKM angeführten Grundstücksnutzungen abgeleitet und analog der aggregierten Typologie des Flächenwidmungsplans zugeordnet werden.

Die Bevölkerungsdaten 2006 wurden auf Gemeindeebene aliquot auf alle Risikoadressen (ausgenommen Widmungskategorie Gewerbe/Industrie), Daten des Kfz-Bestandes 2006 auf Bezirksebene auf alle global vorhandenen Risikoadressen in den entsprechenden Bezirken aufgeteilt (Datenquelle: Statistik Austria). Die Daten der Wohneinheiten zur Lokalisierung und Quantifizierung des Inventars resultieren aus dem Bevölkerungs-Wohnungsverhältnis der Gebäude- und Wohnungszählung 2001 (Statistik AUSTRIa 2004), das auf die Risikoadressen übertragen wurde.

\subsection{Ermittlung repräsentativer monetärer Werte}

Die Analyse wurde mit anonymisierten Einzelpolicen der Tiroler Versicherung durchgeführt. Bei der Geokodierung der Policen ist eine eindeutige Zuweisung der Policenadressen an die Adressen eines Adressbestandsystems (hier: Risikoadressen) und der diesem System immanenten Adresssystematik unerlässlich. Hierbei können zwei grundsätzliche Schritte unterschieden werden: (1) eine strukturelle (Untergliederung der Anschrift in die Variablenfelder Gemeindename, Straßenname und Hausnummer analog zum Referenzsystem) sowie (2) eine inhaltliche (einheitliche, konsistente Schreibweise und Struktur der einzelnen Variablen) Homogenisierung der Daten. Für die beiden Datensätze wurde eine Identifikationsvariable bestehend aus den Primärschlüsseln des Gemeindenamens und des zulässigen Straßennamens sowie der Hausnummer erstellt, so dass $92 \%$ der Policen auf Basis der Risikoadressen verortet werden konnten.

Um neben dem entsprechenden Verwendungszweck der Grundflächen strukturelle räumliche Unterschiede zu berücksichtigen, wurden Raumeinheiten mit homogen angenommenen Siedlungs- und Bevölkerungsstrukturen ausgewiesen. Die Basis der mehrgliedrigen Differenzierung bilden NUTS-3 (Nomenclature des unités territoriales statistiques) Bezugseinheiten. Innerhalb dieser Einheiten wird eine weitere Unterscheidung nach dem durchschnittlichen Nettolohn 2001 aus unselbständiger Arbeit (vgl. Strasser 2001) (Datenquelle: Landesstatistik Oberösterreich) in den einzelnen Gemeinden durchgeführt und zu folgenden Klassen zusammen gefasst: $(1)<€ 13.000,(2) € 13.000$ bis 15.000 und $(3)>€ 15.000$. Sofern Gemeinden eine hohe (200-400) bzw. sehr hohe (>400) Tourismusintensität (Übernachtungen je Einwohner) aufweisen, wurden diese unabhängig der NUTS 3-Zugehörigkeit in diesen zwei Kategorien analysiert, um speziell in diesen Tourismusgemeinden zu verzeichnende Strukturen und hohe Einkommen aus selbständiger Arbeit 


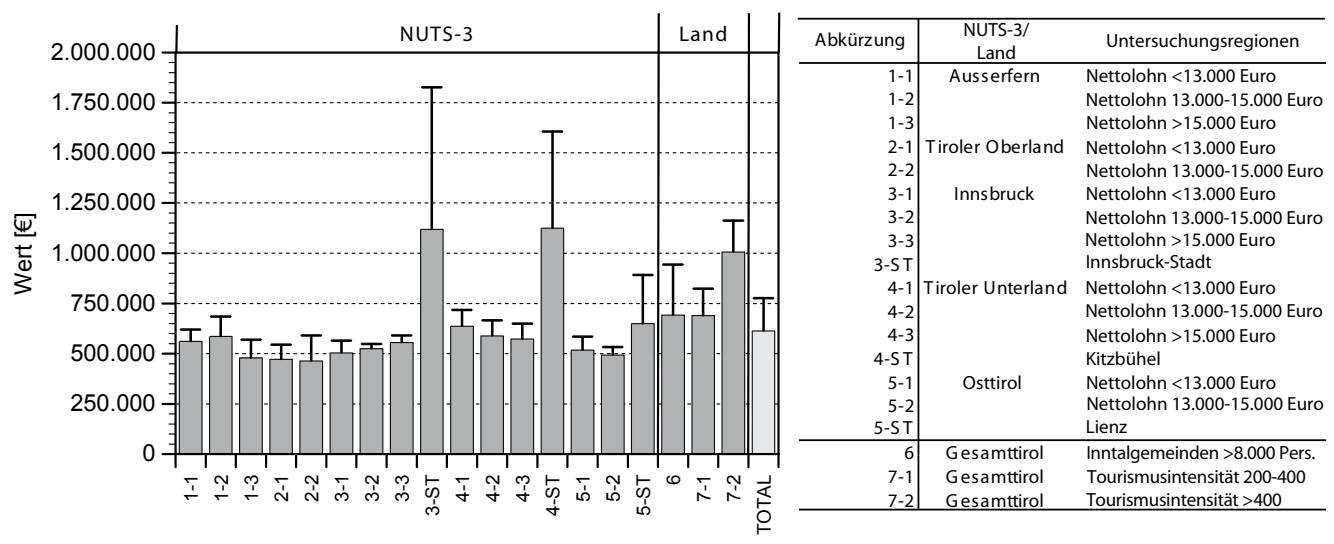

Abb.1: Differenz zwischen durchschnittlichen Gebäudewerten vor (positive Abweichungen) und nach (Balken) der häufigkeitsstatistischen Bearbeitung

Differences in the average values of buildings before (positive variation) and after (bars) statistical frequency processing

Différence entre les valeurs moyennes des bâtiments avant (variations positives) et après (barres) le traitement statistique de fréquence

zu berücksichtigen. Gemeinden mit mehr als 8.000 Einwohnern werden separat in den Kategorien Inntalgemeinden, Innsbruck, Kitzbühel und Lienz betrachtet (siehe auch Abb.1).

Um die Wirkung der Inflation zwischen dem angeführten Nennwert der Policen (Versicherungssummen) zum Zeitpunkt des Vertragsabschlusses und dem realen Wert zum Referenzzeitpunkt mit einbeziehen zu können, mussten die entsprechenden Werte aus den Policen über geeignete Preisindizes an reale Größen angepasst werden. Hierbei empfiehlt der Österreichische Versicherungsverband (VVO) den Verbraucherpreisindex bei der Hausrats- und Lebensversicherung sowie den Baukostenindex bei der Gebäudeversicherung (VVO 2007) (Datenquelle: Statistik Austria). Die Berechnung des Zeitwertes für Kfz erfolgt gemäß der Leasingstaffel KBX2 nach Art. 5 der Allgemeinen Bedingungen für die Kraftfahrzeug-Kaskoversicherung 2006 (Tiroler Versicherung 2007) (Datenquelle: Tiroler Versicherung).

Bei einzelnen Versicherungspolicen von Gebietskörperschaften, Wohnbauträgern o.ä. kann zumeist nicht zwischen Inkasso- und Risikoadresse unterschieden werden (vgl. auch WeIMIN 2001), was zu einer nicht realen Werteakkumulation führt. Diametral hierzu besteht Versicherungsschutz für Objekte mit geringen Versicherungssummen, die nicht Bestandteil der Untersuchung sind. Um derartige Diskrepanzen in den Datenkollektiven systematisch und transparent zu minimieren, wurde mit dem Streuungsmaß des Perzentils für die einzelnen Datenkollektive bei (1) einem Stichprobenumfang $\geq 100$ bzw. bei (2) Stichprobenumfängen zwischen 30 und $<100$ entsprechende Abgrenzungskriterien definiert. Daten, die unter- bzw. oberhalb des Abgrenzungskriteriums lagen, wurden fortan nicht mehr berücksichtigt. Alle Stichprobenumfänge $<30$ gelten als nicht repräsentativ und werden in einer übergeordneten Raumeinheit auf Basis der NUTS 3Region ausgewertet. Abb. 1 verdeutlicht die Ergebnisse dieses Vorgehens auf einer aggregierten Ebene.

Die Versicherungspolicen für die Kategorien Gebäude und Inventar konnten zunächst entsprechend der Differenzierung in Untersuchungsregionen statistisch deskriptiv analysiert (Abb. 2) und weiterführend analog der typisierten Widmungskategorien detaillierter ausgewertet werden (Abb. 3). Aufgrund des partiell geringeren Stichprobenumfangs für die Kategorie Gewerbe/Industrie in den Untersuchungsregionen erfolgte diese Auswertung teilweise auf einer analog zur angeführten häufigkeitsstatistischen Bearbeitung übergeordneten Ebene. Im Rahmen von gewerblichen Gebäudeversicherungen (Gewerbe/Industrie) sind die Inventarwerte dabei bereits in diese eingeschlossen. Werte innerhalb von Tourismusgebieten (Betriebe mit mehr als 150 Betten oder mehr als 75 Räumen) werden für das gesamte Untersuchungsgebiet pauschal betrachtet. Abb. 3 zeigt exemplarisch die ermittelten durchschnittlichen Werte für die Gemeinden im Tiroler Unterland, die 


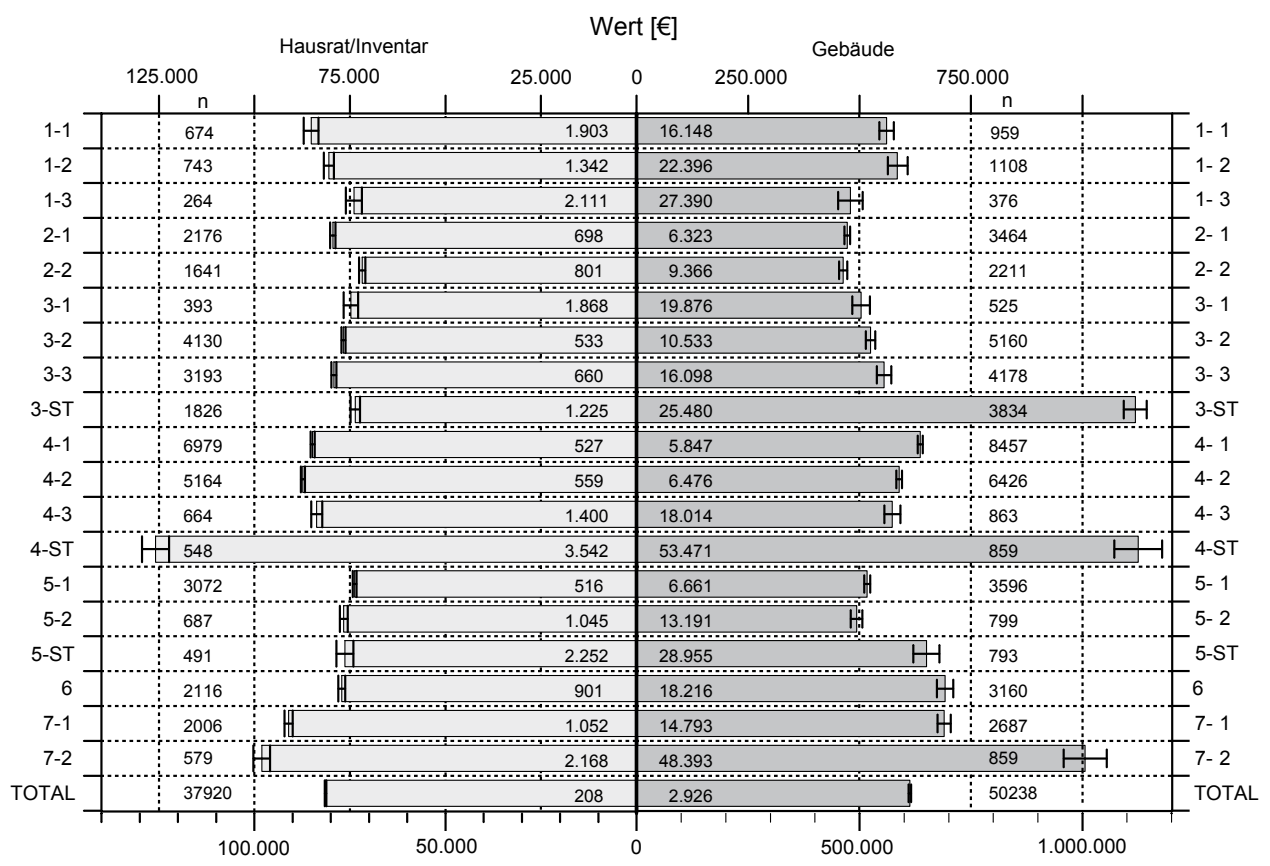

Abb. 2: Allgemeine durchschnittliche Gebäude- und Inventarwerte mit Stichprobengrösse und Standardfehler des Mittelwertes

General average values of buildings and inventory with sample size and standard error of the mean

Valeurs générales moyennes des bâtiments et inventaire avec taille des échantillons et erreur standard de la valeur moyenne

einen durchschnittlichen Pro-Kopf-Nettolohn von $€ 13.000$ bis $€ 15.000$ aufwiesen. Die verorteten Kfzund Lebensversicherungspolicen wurden allgemein auf Bezirksebene ausgewertet.

\subsection{Monetarisierung der Risikoelemente}

Durch relationale Verknüpfungen zwischen den in 2.1. erläuterten Quantifizierungen und Charakterisierungen der einzelnen Risikoelemente und den in 2.2. ermittelten durchschnittlichen Versicherungssummen können den einzelnen Risikoelementen absolute Werte zugewiesen werden.

\section{Ergebnisse}

\subsection{Mittlere Versicherungssummen}

Die dargestellte Methode ermöglicht eine indizierte und der Bearbeitungstiefe angepasste, repräsentative Ausweisung von Objektwerten. In einer ersten Dimension können bei der Betrachtung von allgemeinen Gebäudeversicherungssummen in Tirol klare Disparitätenmuster erkannt werden. Die Summen in urba- nen und touristisch geprägten Räumen liegen hierbei deutlich über dem Mittel, im ländlichen Raum ist tendenziell - mit Ausnahme des suburbanen Umlandes von Innsbruck - ein inverses Verhältnis zum NettoEinkommen zu beobachten. Diese Struktur ist bei Inventarsummen nicht derart ausgeprägt, vielmehr kann in urbanen Räumen ein geringerer Wert konstatiert werden. Hinsichtlich der durchschnittlichen Versicherungssumme für Gebäude und Inventar nimmt die Gemeinde Kitzbühel eine Sonderstellung ein (vgl. Abb. 2). Bei der Betrachtung in einer nach der Widmung differenzierten zweiten Dimension sind große Unterschiede in den Gebäudewerten innerhalb einer Region ersichtlich, die Inventarwerte hingegen bewegen sich in einer geringen Spannbreite; Ausnahmen bilden Wohnungen in Tourismusgebieten. Die durchschnittlichen Werte der Kategorie Gewerbe/Industrie besitzen eine weite Spannbreite von $-63 \%$ bis $+157 \%$ um den Mittelwert von $€ 1,8$ Mio. mit den höchsten Werten im mittleren und östlichen Inntal und tendenziell geringeren Werten in der Peripherie. Die Kfz-Werte bewegen sich mit geringen Schwankungsbreiten um einen zentralen Mittelwert, bei Lebens- 


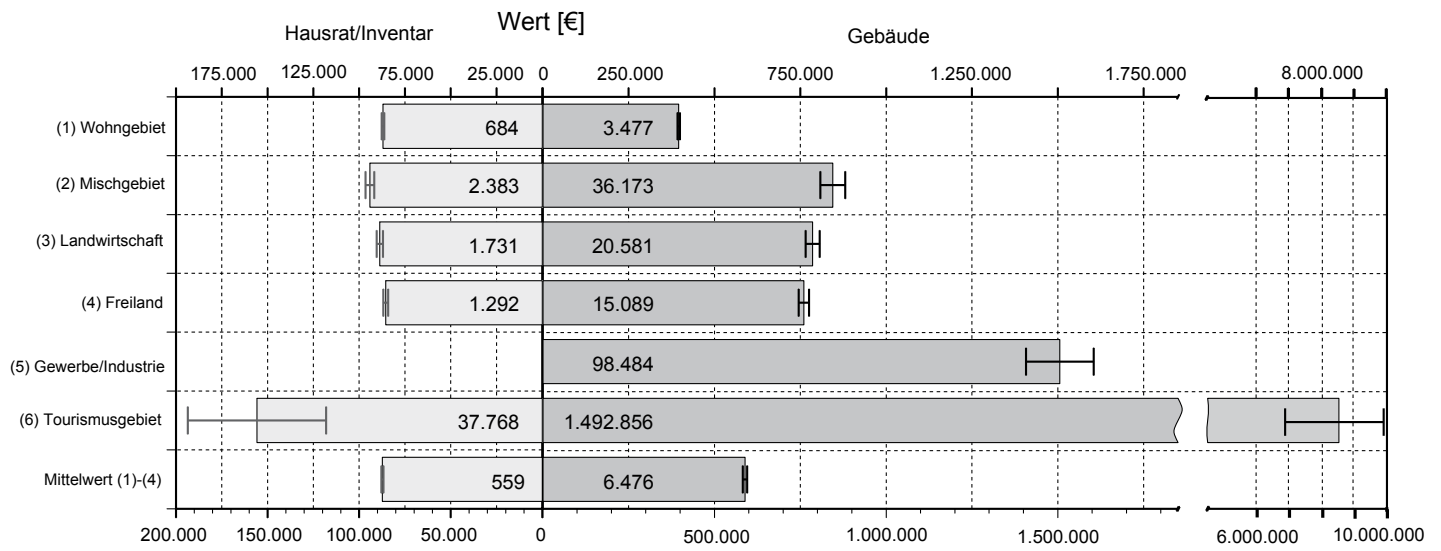

Abb. 3: Statistische Auswertung der differenzierten mittleren Versicherungssumme für Gebäude und Inventar in der Untersuchungsregion Tiroler Unterland; Nettoeinkommen $€$ 13.000-15.000 mit dem Standardfehler des Mittelwertes

Statistical evaluation of the differentiated average insurance sum for buildings and inventory within the study area Tiroler Unterland; net income $€ 13.000-15.000$ and the standard error of the mean

Evaluation statistique des montants d'assurance moyens différenciés selon les bâtiments et inventaire dans la région d'étude de l'Unterland tyrolien; revenu net de €13'000-15'000 avec erreur standard de la valeur moyenne

versicherungen ist tendenziell ein West-Ost-Gefälle erkennbar (vgl. Tab.1).

\subsection{Monetarisierte Risikoelemente}

Den einzelnen Risikoelementen können repräsentative monetäre Werte zugewiesen werden, die sich kumulativ für das Untersuchungsbiet darstellen lassen und somit eine monetäre Bewertung des vorhandenen Werteinventars in Tirol ermöglichen (vgl. Tab.1). Hierbei wird einerseits zwischen Risikoadressen und -gebäuden und andererseits zwischen reinen Gebäudeund Inventarwerten sowie integralen Werten unterschieden. Es kann verdeutlicht werden, dass v.a. im urbanen Raum große Disparitäten zwischen den ermittelten Werten für Risikoadressen und Risikogebäuden (mehrere Risikoadressen bei einem Risikogebäude möglich) bestehen. Abb. 4 zeigt, dass sich die höchsten durchschnittlichen Einzelwerte für Gebäude und Inventar in Innsbruck, Kitzbühel und stark touristisch geprägten Gemeinden befinden, wogegen in ländlich geprägten Gemeinden die niedrigsten Einzelwerte zu verzeichnen sind.

\subsection{Datenbank}

Für eine effiziente und nachhaltige Nutzung der vorgestellten Daten und Ergebnisse im Rahmen von Risikoanalysen wurde eine entsprechende Geodatenbankstruktur implementiert. Effizient bedeutet hierbei, dass Geometriedaten in einer Geodatenbank
(GDB), Sachdaten primär in einer mit der GDB verbundenen relationalen Datenbank und sekundär als Tabelle in der GDB geführt und über individuell mit Regeln definierte Relationen mit den Geometriedaten verbunden werden. Topologieregeln garantieren entsprechend den vom Maßstab abhängigen Notwendigkeiten die Integrität der Geometriedaten zueinander. Eine nachhaltige Nutzung beinhaltet die Möglichkeit, Geo- und Sachdaten kontinuierlich an dafür geeigneten Schnittstellen aktualisieren (z.B. Dynamik der Kulturlandschaft, Indexanpassung) sowie neue Daten integrieren zu können. Abb. 5 zeigt exemplarisch einen visualisierten Ausschnitt aus der Datenbank.

\section{Diskussion und Fazit}

Grundsätzlich ist ein vergleichender Diskurs mit Ergebnissen weiterer Studien nur relativ schwer möglich, da die Resultate wesentlich von den zur Verfügung stehenden Basisdaten abhängen. Darüber hinaus bestehen substanzielle Unterschiede hinsichtlich sozialer, wirtschaftlicher sowie kultureller Strukturen in unterschiedlichen Untersuchungsgebieten. Nur in wenigen Studien werden explizite, in der Regel kumulative Gebäudewerte für ein Untersuchungsgebiet publiziert. Kfz-Preise variieren im internationalen Vergleich stark und können demnach länderübergreifend nur mit Vorbehalt verglichen werden. 


\begin{tabular}{|c|c|c|c|c|c|c|c|c|c|c|}
\hline \multirow[b]{2}{*}{ Bezirke } & \multicolumn{2}{|c|}{ Leben } & \multicolumn{2}{|c|}{ KFZ-Haftpflicht } & \multicolumn{3}{|c|}{$\begin{array}{l}\text { Risikoadressen - gesamt } \\
\text { (Gebäude, Inventar) }\end{array}$} & \multicolumn{3}{|c|}{$\begin{array}{l}\text { Risikoadressen - Wohnbevölkerung } \\
\text { (Gebäude, Inventar) }\end{array}$} \\
\hline & Bevölkerung & Summe & KFZ & Summe & Adresspunkte & Summe & Mittelwert & Adresspunkte & Summe & Mittelwert \\
\hline Reutte & 31.918 & 1,126 Mrd. & 22.735 & 378 Mio. & 9.983 & 7,284 Mrd. & 729.631 & 9655 & 6,639 Mrd. & 687.647 \\
\hline Landeck & 45.021 & 1,725 Mrd. & 30.410 & 546 Mio. & 11.834 & 8,592 Mrd. & 726.055 & 11325 & 7,777 Mrd. & 686.730 \\
\hline Imst & 55.096 & 1,735 Mrd. & 38.289 & 572 Mio. & 16.076 & 10,867 Mrd. & 675.995 & 15326 & 9,442 Mrd. & 616.031 \\
\hline Innsbruck Stadt & 116.881 & 3,232 Mrd. & 65.044 & 1,055 Mrd. & 12.432 & 19,216 Mrd. & 1.545 .657 & 12140 & 19,027 Mrd & 1.567 .265 \\
\hline Innsbruck Land & 161.341 & 5,443 Mrd. & 104.158 & 1,616 Mrd. & 38.498 & 26,775 Mrd. & 695.499 & 37189 & 24,272 Mrd. & 652.675 \\
\hline Schwaz & 77.562 & 1,503 Mrd. & 50.915 & 824 Mio. & 19.885 & 16,075 Mrd. & 808.381 & 18949 & 14,586 Mrd. & 769.735 \\
\hline Kufstein & 97.773 & 2,155 Mrd. & 62.774 & 983 Mio. & 25.753 & 19,364 Mrd. & 751.919 & 24625 & 17,489 Mrd. & 710.202 \\
\hline Kitzbühel & 61.222 & 1,834 Mrd. & 40.633 & 654 Mio. & 20.604 & 17,943 Mrd. & 870.852 & 19719 & 16,805 Mrd. & 852.229 \\
\hline Lienz & 50.572 & 1,367 Mrd. & 31.907 & 546 Mio. & 12.956 & 8,721 Mrd. & 673.088 & 12334 & 7,698 Mrd. & 624.134 \\
\hline Tirol & $697.386 \quad 2$ & 20,123 Mrd. & 446.865 & 7,173 Mrd. & 168.021 & 134,837 Mrd. & 802.499 & $162.262 \quad 12$ & $123,734 \mathrm{Mrd}$ & 62.559 \\
\hline
\end{tabular}

\begin{tabular}{|l|rrrr|rr|}
\hline & \multicolumn{4}{|c|}{ Risikogebäude } & (Gebäude, Inventar) & \multicolumn{2}{c|}{$\begin{array}{c}\text { Summe Risikoadressen } \\
\text { (Gebäude, Inventar, KFZ, Leben) }\end{array}$} \\
\hline Bezirke & Gebäude & $\varnothing$ Fläche $\left(\mathrm{m}^{2}\right)$ & \multicolumn{1}{c}{ Summe } & Mittelwert & \multicolumn{1}{c|}{ Summe } & Mittelwert \\
\hline Reutte & 9.260 & 269,8 & $7,284 \mathrm{Mrd}$. & 786.599 & $8,788 \mathrm{Mrd}$. & 925.516 \\
Landeck & 10.475 & 311,0 & $8,592 \mathrm{Mrd}$. & 820.252 & $10,862 \mathrm{Mrd}$. & 917.896 \\
Imst & 14.774 & 298,3 & $10,867 \mathrm{Mrd}$. & 735.569 & $13,174 \mathrm{Mrd}$. & 819.496 \\
Innsbruck Stadt & 7.466 & 606,0 & $19,216 \mathrm{Mrd}$. & 2.573 .632 & $23,502 \mathrm{Mrd}$. & 1.890 .439 \\
Innsbruck Land & 32.781 & 313,9 & $26,775 \mathrm{Mrd}$. & 816.820 & $33,835 \mathrm{Mrd}$. & 878.865 \\
Schwaz & 17.968 & 312,0 & $16,075 \mathrm{Mrd}$. & 894.626 & $18,404 \mathrm{Mrd}$. & 880.287 \\
Kufstein & 23.778 & 296,4 & $19,364 \mathrm{Mrd}$. & 814.337 & $22,501 \mathrm{Mrd}$. & 873.737 \\
Kitzbühel & 19.147 & 292,8 & $17,943 \mathrm{Mrd}$. & 937.166 & $20,432 \mathrm{Mrd}$. & 991.639 \\
Lienz & 11.483 & 282,1 & $8,721 \mathrm{Mrd}$. & 759.430 & $10,634 \mathrm{Mrd}$. & 820.766 \\
\hline Tirol & 147.132 & 315,9 & $134,837 \mathrm{Mrd}$. & 916.433 & $162,132 \mathrm{Mrd}$. & 964.949 \\
\hline
\end{tabular}

Tab. 1: Überblicksdarstellung der Ergebnisse gemittelt auf Bezirksebene

Overview of the average results at district level

Présentation générale des résultats moyens par district

Im Untersuchungsgebiet ermittelte KEILER (2004) mit einer Lokalanalyse in Galtür für das Jahr 2000 einen durchschnittlichen Gebäudewert (inklusive Inventar) von $€ 916.637$, was einem über den Baukostenindex angepassten Wert für 2006 von $€ 1.111 .562$ entspricht. Der im Rahmen dieser Regionalstudie für Galtür ermittelte Wert für eine durchschnittliche Risikoadresse beträgt $€ 1.183 .068$ (vgl. Abb. 4). Dies ist ein Indikator, der die Aussage zulässt, dass die von Hamann \& Reese (2000) sowie Reese \& Markau (2004) für ein norddeutsches Untersuchungsgebiet diskutierten beachtlichen Differenzen in Abhängigkeit von der Maßstabsebene durch die vorgestellte Methodik und die daraus resultierenden Ergebnisse verhindert werden können. Somit wird eine adäquate Darstellung der monetarisierten Gebäude- und Inventarwerte für regionale Studien möglich. Für KfzWerte ist entsprechend der Methodik keine Studie mit Vergleichsmöglichkeiten bekannt. Die vorgenommene Bewertung menschlichen Lebens verfolgt einen rein versicherungstechnischen Ansatz (durchschnittliche Versicherungssummen aus Lebensversicherungen), ein Vergleich mit anderen Studien ist nicht möglich.

Es kann gezeigt werden, dass eine klare Deklaration der Objektdefinition bzw. der Objektabgrenzung (Risikoadresse versus -gebäude) essentiell ist - dies auch unter dem Blickwinkel der Überführung von ermittelten Werten auf weitere Studien und der Anwendung von Anfälligkeitsfunktionen als Vulnerabilitätsfaktor bei Folgenanalysen (Merz 2006). Allgemein ist die Differenzierung von finanziellen und ökonomischen Werten für die Aussagekraft und Interpretation relevant. Nach Merz (2006) liegen die Differenzfaktoren für Immobilien typischerweise in einer Größenordnung um den Wert 2.

Die Höhe des Werteinventars einer Raumeinheit wird (1) durch den (durchschnittlichen) Wert sowie (2) durch die räumliche Konzentration der Risikoelemente definiert. Folglich ist eine aktuelle und präzise Lokalisierung, Quantifizierung und Charakterisierung der Risikoelemente sowie die Verwendung repräsentativer monetärer Werte maßgebend für die Qualität derartiger Studien.

Durch die vorgestellte Arbeit konnte für Tirol eine homogene Datenbasis geschaffen werden. Diese ermöglicht zukünftig im Rahmen von konkret durchzuführenden Risikoanalysen eine effiziente Schadenspotenzialermittlung durch Expositions- bzw. 


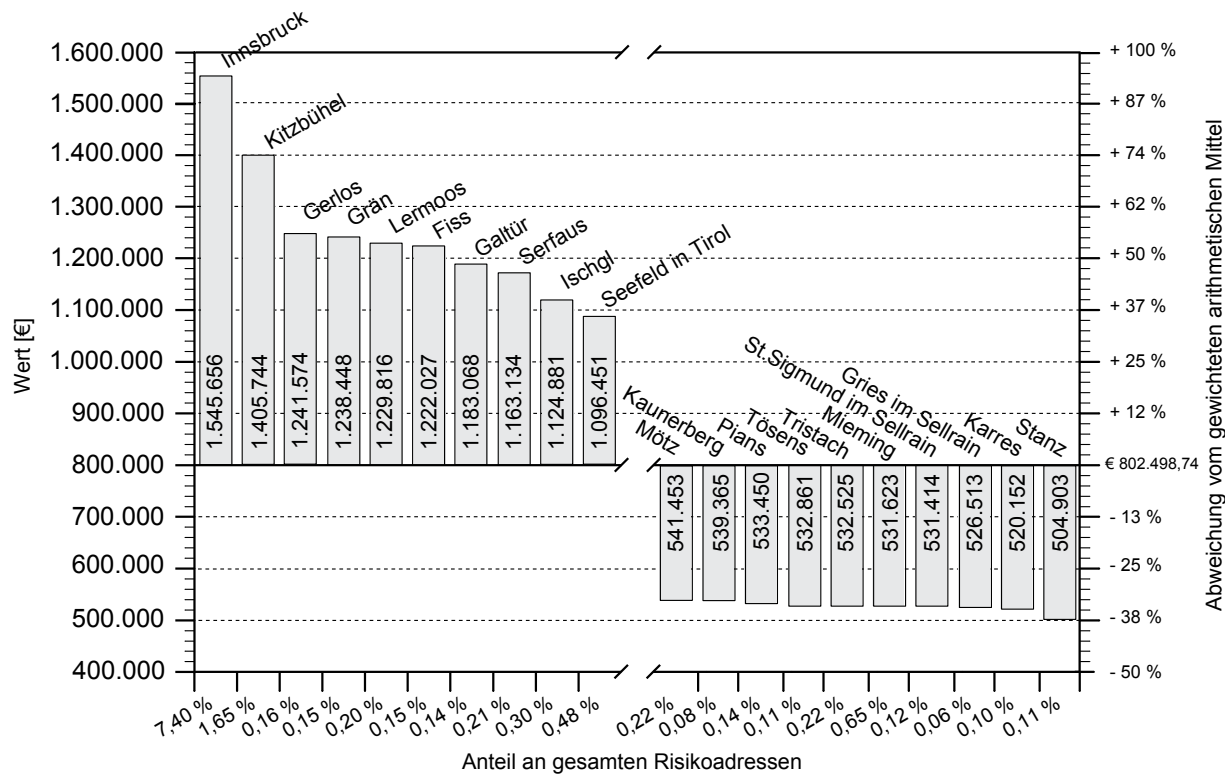

Abb. 4: Disparitätendiagramm: Gemeinden mit den durchschnittlich höchsten bzw. niedrigsten Werten für allgemeine Risikoadressen (Gebäude, Inventar)

Diagramme of disparities: communities with the highest, respectively lowest, average values for general risk addresses (buildings, inventory)

Diagramme des disparités: communes présentant les valeurs les plus élevées et les plus basses pour des adressesrisque générales (bâtiments, inventaire)

Schadensausmaßberechnung mittels Folgenanalysen (vgl. Borter 1999; Heinimann et al. 1998; HollenSTEIN 1997) für regionale Untersuchungen. Darüber hinaus entstand eine wirtschaftliche Datenbasis (Heinimann et al. 1998; Kienholz 2005) für lokale Untersuchungen, die das Postulat der sachlichen Richtigkeit und Nachvollziehbarkeit (HeInimanN et al 1998; Kienholz 2005) verfolgt. Des weiteren wird eine entsprechende Methode aufgezeigt, welche die eingangs erwähnten Differenzfaktoren und somit ein wesentliches Unterschätzen der vorhandenen Werte auf der regionalen Maßstabsebene verhindern, was sich entsprechend auf die Qualität und Aussagekraft der auf diesen Arbeiten aufbauenden risikobasierten Analysen auswirkt.

\section{Dank}

Die vorgestellte Studie ist Bestandteil eines Forschungsprojektes zur Risikoanalyse extremer Naturgefahren am alpS Zentrum für Naturgefahren Management in Innsbruck und wird in Kooperation mit und Unterstützung durch die Tiroler Versicherung und des Bundeslandes Tirol durchgeführt.

\section{Literatur}

Bohle, H.-G. \& T. Glade (2007): Vulnerabilitätskonzepte in Sozial- und Naturwissenschaften. - In: FELGENTREFF, C. \& T. Glade (Hrsg.): Naturrisiken und Sozialkatastrophen. - = Heidelberg: Spektrum Akademischer Verlag: 99-119.

BORTER, P. (1999): Risikoanalyse bei gravitativen Naturgefahren. Methode. - = Umwelt-Materialien 107/I, Bern: 1-115.

Bundesamt fÜr WASSER UND Geologie (BWG) (2005): Priorisierungsverfahren für Hochwasserschutzprojekte. Abschätzung des Schadenpotenzials Überschwemmung und Übermurung. - http://www. bafu.admin.ch/naturgefahren/01923/01954/index. html?lang=de 08.10.2007.

BUND DER INGENIEURE FÜR WASSERWIRTSCHAFT, ABFALLWIRTSCHAFT UND KULTURBAU (BWK) E.V. (Hrsg.) (2001): Hochwasserschadenspotenziale. $-=$ BWK-Berichte 1/2001, Düsseldorf: 1-40.

Douglas, J. (2007): Physical vulnerability modelling in natural hazard risk assessment. - In: Natural hazards and earth system sciences 7, 2:283-288.

Fuchs, S., BRÜNDL, M. \& J. STÖTter (2004): Development of avalanche risk between 1950 and 2000 in the 


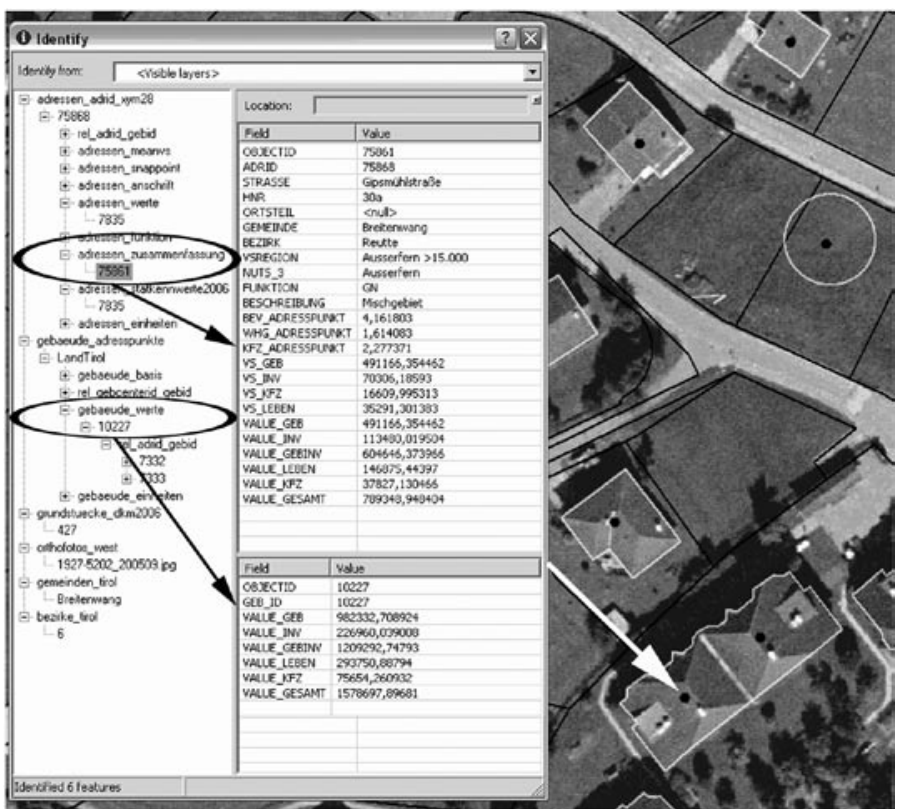

Abb. 5: Visualisierter Datenbankausschnitt für eine Risikoadresse bzw. ein Risikogebäude Visualised database extract for a single risk address, respectively for a single risk building

Extrait visuel d'une adresse-risque et d'un bâtiment-risque de la base de données municipality of Davos, Switzerland. - In: Natural hazards and earth system sciences 4, 2: 263-275.

Fuchs, S. \& M.C. McAlpin (2005): The net benefit of public expenditures on avalanche defence structures in the municipality of Davos, Switzerland. - In: Natural hazards and earth system sciences 5, 3: 310-330.

Gewalt, M., Klaus, J., Peerbolte, E.B., Pflügner, W., SchmidtKe, R.F. \& L. Verhagen (1996): EUROflood technical annex 8. Economic assessment of flood hazards. Regional scale analysis decision support system (RSA-DSS). - München: 1-37.

Glade, T. \& M. Crozier (2005): A review of scale dependency in landslide hazard and risk analysis. - In: Glade, T., Anderson, M. \& M. Crozier (eds): Landslide hazard and risk. - = Chichester: Wiley: 75-138.

Hamann, M. \& S. ReEse (2000): Wertermittlung und Schadensanalyse als Instrument für die Küstenschutzplanung in Schleswig-Holstein. - In: Bremer Beiträge zur Geographie und Raumplanung 36: 139-147.

Heinimann, H.R., Hollenstein, K., Kienholz, H. \& B. Krummenacher (1998): Methoden zur Analyse und Bewertung von Naturgefahren. - = Umwelt-Materialien 85, Bern: 1-248.

Hollenstein, K. (1997): Analyse, Bewertung und Management von Naturrisiken. - Zürich: vdf Hochschulverlag an der ETH Zürich.

IKoNE - INTEGRIERENDE KONZEPTION NECKAR-EINZUGSGEBIET (2003): Integrierte Konzeption NeckarEinzugsgebiet. Grundlagen und vereinfachtes Verfahren zur flächenhaften Ermittlung von Hochwasserschadenpotentialen. Stand April 2003. - http://
www.ikone-online.de/fileadmin/template/hefte/SchadenpotentialeIKoNE.pdf 11.06.2007.

KeILER, M. (2004): Development of the damage potential resulting from avalanche risk in the period 19502000, case study Galtür. - In: Natural hazards and earth system sciences 4, 2: 249-256.

Keiler, M., Meissl, G. \& J. Stötter (2004): Determination of the damage potential. A contribution to the analysis of avalanche risk. - In: BREBBIA, C. (ed.): Risk analysis IV. - = WIT transactions on ecology and the environment 67, Southampton: 187-196.

KeIler, M., ZischG, A. \& S. Fuchs (2006): Methoden zur GIS-basierten Erhebung des Schadenpotenzials für naturgefahreninduzierte Risiken. - In: Strobl, J. \& C. Roth (Hrsg.): GIS und Sicherheitsmanagement. - Heidelberg: Wichmann:118-128.

Kienholz, H. (2005): Analyse und Bewertung alpiner Naturgefahren. Eine Daueraufgabe im Rahmen des integralen Risikomanagements. - In: Geographica Helvetica $60,1: 3-15$.

Kleist, L., Thieken, A.H., Köhler, P., Müller, L., SeIFERT, I., Borst, D. \& U. Werner (2006): Estimation of the regional stock of residential buildings as a basis for a comparative risk assessment in Germany. - In: Natural hazards and earth system sciences 6, 4: 541-552.

KoK, M., Huizinga, H.J., Vrouwenvelder, A.C.W.M. \& A. Barendregt (2004): Standaardmethode 2004. Schade en Slachtoffers als gevolg van overstromingen. - http://www.verkeerenwaterstaat.nl/kennisplein/ uploaded/DWW/2005-04/269457/DWW-2005-005\%20 Rapport\%20Standaardm_e-book.pdf 11.06.2007. 
LeITER, A.M. (2006): Monetary risk valuation in context with natural hazards. - Dissertation am Institut für Wirtschaftstheorie, -politik und -geschichte der Universität Innsbruck.

Merz, B. (2006): Hochwasserrisiken. Grenzen und Möglichkeiten der Risikoabschätzung. - Stuttgart: E. Schweizerbart'sche Verlagsbuchhandlung.

MeYER, V. (2005): Methoden der Sturmflut-Schadenpotenzialanalyse an der deutschen Nordseeküste. - http:// www.ufz.de/data/ufzdiss_03_2005_optimiert4200.pdf 12.03.2007.

Ministerium FÜr UMWELt, RAUMORDNUNG UND LANDWIRTSCHAFT NORDRHEIN-WESTFALEN (MURL) (2000): Potentielle Hochwasserschäden am Rhein in NRW. - Düsseldorf: Ministerium für Umwelt, Raumordnung und Landwirtschaft Nordrhein-Westfalen: 1-32.

ReEse, S. \& H.-J. MarkaU (2004): Mikroskalige Wertermittlung in sturmgefährdeten Küstenniederungen. - In: Jahrbuch der Hafenbautechnischen Gesellschaft; http://www.sterr.geographie.uni-kiel.de/mare/htg.pdf 12.03.2007.

StatistiK Austria (Hrsg.) (2004): Gebäude- und Wohnungszählung 2001. Hauptergebnis Tirol. - Wien: Verlag Österreich GmbH.

Strasser, R. (2001): Dokumentation zur Abgabenberechnung auf unselbständige Erwerbseinkommen 2001.

- http://tirolatlas.uibk.ac.at/topics/population/income/ dokumentationabgabenquote.pdf 12.09.2007.

Tiroler Versicherung (2007): Berechnung des KfzZeitwertes. - Schriftliche Mitteilung v. 08.11.2007.

VERSICHERUNGSVERBAND ÖSTERREICH (VVO) (2007): Indizes Empfehlungen in der Sachversicherung.-Schriftliche Mitteilung v. 12.04.2007.

WeIMIN, D. (2001): Building a more profitable portfolio. Modern portfolio theory with application to catastrophe insurance. - London: Reactions Publishing Group. WiLhelm, C. (1997): Wirtschaftlichkeit im Lawinenschutz. Methodik und Erhebung zur Beurteilung von Schutzmassnahmen mittels quantitativer Risikoanalyse und ökonomischer Bewertung. $-=$ Mitteilungen des Eidgenössischen Instituts für Schnee- und Lawinenforschung 54, Davos: 1-309.

\section{Zusammenfassung: Ermittlung des monetären Werte- inventars als Basis von Analysen naturgefahrenindu- zierter Risiken in Tirol (Österreich)}

Im Rahmen von Risikoanalysen besitzt die Quantifizierung und Monetarisierung der potenziellen Risikoelemente zur Ermittlung des Werteinventars eine wesentliche Rolle, speziell bei regionalen Maßstabsebenen bestehen jedoch große Unsicherheiten. Durch eine differenzierte Bearbeitung und die Anwendung von repräsentativen, aus einzelnen Versicherungspolicen analysierten Werten können diese Unsicherheiten wesentlich minimiert werden. Eingebunden in eine moderne (Geo-)Datenbankstruktur entsteht ein effi- zientes Instrument für Expositions- und Folgenanalysen naturgefahreninduzierter Risiken.

Schlüsselwörter: Risikoanalyse, Risikoelemente, monetäres Werteinventar, Versicherungssummen, Datenbank

Summary: Monetary value inventory as basis for risk analysis of natural hazards in Tyrol, Austria

The quantification and monetary valuation of potential elements at risk plays a major role within the framework of risk analysis. However, especially at a regional scale, large uncertainties exist. A considerable reduction of these uncertainties can be achieved by a differentiated processing and application of representative values taken from the analysis of individual insurance contracts. Their integration in a (geo)database structure allows for an efficient instrument suitable for the analysis of exposition and the consequences of risks due to natural hazard processes.

Keywords: risk analysis, elements at risk, monetary value inventory, insurance values, database

\section{Résumé: Détermination de la valeur d'inventaire comme base d'analyse des risques naturels dans le Tyrol (Autriche)}

Dans le cadre d'une analyse des risques, la quantification et la monétarisation des éléments-risques potentiels nécessaires à la détermination de l'inventaire des valeurs jouent un rôle essentiel, bien qu'il existe de grandes incertitudes notamment à l'échelle régionale. Il est possible de réduire sensiblement ces incertitudes au moyen d'un traitement différencié et de valeurs représentatives découlant de l'analyse des polices d'assurance individuelles. L'intégration dans une base de données géoréférencée permet de créer un instrument efficace pour l'analyse de l'exposition aux risques induits par les dangers naturels et de leurs conséquences.

Mots-clés: analyse du risque, éléments-risques, inventaire des valeurs monétaires, montants d'assurance, base de données

Mag. rer. nat. Matthias Huttenlau, alpS Zentrum für Naturgefahren Management Innsbruck, Grabenweg 3, A-6020 Innsbruck, sowie Institut für Geographie der Leopold-Franzens-Universität Innsbruck, Innrain 52, A-6020 Innsbruck, Österreich.

e-mail: matthias.huttenlau@uibk.ac.at

Prof. Dr. Johann Stötter, Institut für Geographie der Leopold-Franzens-Universität Innsbruck, Innrain 52, A-6020 Innsbruck, Österreich.

e-mail: hans.stoetter@uibk.ac.at

\section{Manuskripteingang/received/manuscrit entré le 14.1.2008}

Annahme zum Druck/accepted for publication/accepté pour l'impression: 17.6.2008 